\title{
Estimation du poids du fotus zébu peulh à partir de mesures échographiques des paramètres fotaux
}

\author{
A.T. Yamboué, M. Zongo, B. Traoré, O. Diallo, and C. Hanzen
}

\begin{abstract}
Résumé : Cette étude vise à estimer le poids du fotus zébu peulh à partir des mesures échographiques des diamètres de l'abdomen (DA), du bipariétal (DBP), du cordon ombilical (DCO), de la corne utérine (DCU) et des longueurs du fémur (LF), du tibia (LT), et du dos (LD). Elle a concerné soixante et un ( $n=61)$ utérus gravides recueillis après abattage de femelles gestantes. Toutes les gestations étaient simples. Les examens ont été réalisés en bain d'eau au moyen d'une sonde convexe de 3,5 MHz. Les utérus ont été ensuite disséqués afin de rapporté le poids des fotus au moyen d'une balance. Les données ont été analysées au moyen du système de régression curvilinéaire. Les formules d'estimation du poids à partir des paramètres biométriques ont été de type $y=a x^{b}$, où $y$ est le poids $(\mathrm{g})$, $a$ et $b$ _constantes, et $x$ est le paramètre biomètrique $(\mathrm{mm})$. Toutes les structures morphologiques étudiées ont présenté des corrélations positives et hautement significatives avec le poids du fotus $(p<0,0001)$. Toutefois, les DA $\left(R^{2}=0,94\right)$, DBP $\left(R^{2}=0,80\right)$, et la $\mathrm{LF}\left(R^{2}=0,81\right)$ semblent être les plus appropriés pour l'estimation du poids du foetus. À défaut de ces paramètres, les autres structures étudiées peuvent servir de repère dans l'estimation du poids.
\end{abstract}

Mots-clés : poids du fotus, mesure échographique, paramètres biométriques, utérus gravide, zébu peulh.

\begin{abstract}
This study aims to estimate fetal weight using ultrasound measurements of abdominal (AD), biparietal (BPD), umbilical cord (UCD), uterine horn (UHD) diameters and femur (FL), tibia (TL), and crown rump (CRL) lengths in zebu peulh. It concerned $61(n=61)$ simple gravid uteri collected from slaughterhouse. Ultrasonographic scanning was carried out in a water bath using a real-time B-mode ultrasound machine having 3.5 $\mathrm{MHz}$ convex probe. The uterus was then dissected to report the weight of the fetuses using a scale. The data were subjected to simple curvilinear regression analysis. The derived weight estimation formulas type was $y=a x^{b}$. Where $y$ is the fetal weight (g), $a$ and $b$ are constants, and $x$ is the biometric parameter $(\mathrm{mm})$. All morphological structures studied showed positive and highly significant correlations with fetal weight $(p<0.0001)$. However, $\mathrm{AD}\left(R^{2}=0.94\right)$, BPD $\left(R^{2}=0.80\right)$, and FL $\left(R^{2}=0.81\right)$ appear to be the most appropriate parameters for estimating fetal weight in zebu peulh. In the absence of these parameters, the other structures studied can be used as a benchmark in weight estimation.
\end{abstract}

Key words: fetal weight, ultrasound measurement, biometric parameters, pregnant uterus, zebu peulh.

\section{Introduction}

En médecine humaine, l'estimation du poids du foetus à la naissance est le facteur déterminant de la survie du nouveau-né (Lafont et al. 2016). Des poids extrêmes faibles ou élevés de fotus à la naissance sont associés à des complications maternelles ou néonatales pendant l'accouchement et le puerperium. L'estimation du poids du fotus est utile dans l'anticipation de la prise en charge néonatale en cas de prématurité, de retard de croissance ou de discordance de croissance (Branum et Schoendorf 2003; Ivars et al. 2010). La discordance ou retard de croissance constitue un facteur de prématurité et de décès néonatal des jumeaux (Lackman et al. 2001; Branum et Schoendorf 2003). De même, les conséquences d'une dystocie des épaules, en cas d'accouchement d'un nouveau-né macrosome ou d'une rétention de la tête dernière par disproportion foeto-pelvienne, peuvent être dramatiques aussi bien pour le nouveau-né (complications

Reçu le 10 janvier 2020. Accepté le 25 mai 2020.

A.T. Yamboué, M. Zongo, B. Traoré, and O. Diallo. Laboratoire de Physiologie animale, Département de Biologie et Physiologie Animale, UFR/SVT, Université Joseph KI-ZERBO, Ouagadougou 03 BP 7021, Burkina Faso.

C. Hanzen. Département de Gestion Vétérinaire des Ressources Animales, Faculté de Médecine Vétérinaire, Université de Liège, B42 Sart Tilman, Liège B - 4000, Belgique.

Auteur correspondant : M. Zongo (courriel : moussa_zongo59@yahoo.fr, moussa_zongo@univ-ouaga.bf).

Les droits d'auteur demeurent la propriété des auteurs ou de leur établissement. Il est possible d'obtenir (gratuitement dans la plupart des cas) l'autorisation de réutiliser du contenu en passant par copyright.com. 
neurologiques centrales ou périphériques) que pour la mère (délabrements périnéaux, hémorragie du post-partum) (Perdriolle-Galet et al. 2014). Il est donc indéniable que l'évaluation du poids fotal occupe une place de choix dans le suivi de la croissance fotale. Elle s'effectue en routine soit par la voie clinique ou par la voie échographique. La méthode échographique qui est la plus utilisée se fonde sur un système d'imagerie et de biométrie fotale. Cette méthode serait la plus indiquée pour l'estimation du poids des fotus (Hadlock et al. 1985; Ivars et al. 2010).

En médecine vétérinaire, l'estimation du poids du fœtus, bien qu'importante, est encore marginale (Azevedo et al. 2007; Carr et al. 2011; Nguyen et al. 2012; Zongo et al. 2014). Elle permet d'identifier les restrictions de croissance foetale qui est l'une des principales causes de complication de la gestation et certaines maladies associées au développement embryonnaire (Carr et al. 2011). Les données sur l'âge, le nombre et le poids du fœtus permettent aux producteurs de regrouper les animaux selon leur besoin nutritionnel et d'organiser un rationnement approprié au cours du dernier trimestre de la gestation (Vural et al. 2008; Craig et al. 2009), de réaliser le tarissement des femelles à des périodes adéquates et de les préparer à la parturition (Robinson 1977). Chez les équins, les travaux se multiplient pour déceler les facteurs susceptibles d'influencer le poids du fotus (Elliott et al. 2009; Vieira et al. 2018). Chez les ovins, caprins tout comme chez les souris, plusieurs formules d'estimations du poids du fotus ont été déjà proposées (Carr et al. 2011; Nguyen et al. 2012; Zongo et al. 2014). Dans l'espèce bovine, la plupart des travaux disponibles sur le contrôle échographique de la reproduction se rapportent à l'activité ovarienne, au diagnostic de gestation, la détermination de l'âge et du sexe des fotus (Tainturier et al. 2000; Tainturier et al. 2004; Pitala et al. 2012). Les études sur l'estimation du poids du foetus ne sont pas encore envisagées.

La présente étude vise à prédire le poids du fœetus intra-utérin ou du nouveau-né à partir de mesures échographiques de paramètres biométriques chez le zébu peulh. Il s'agira d'établir une formule d'estimation du poids du fotus à partir des dimensions échographiques des diamètres de l'abdomen (DA), du bipariétal (DBP), du cordon ombilical (DCO), de la corne utérine (DCU) et les longueurs du dos (LD), du fémur (LF) et du tibia (LT).

\section{Matériels et Méthodes}

\section{Animaux}

Cette étude a concerné soixante-un (61) utérus gravides de femelles zébus toutes venant à l'abattoir frigorifique de Ouagadougou. Le poids et l'âge des femelles à l'abattage ont été respectivement compris dans les intervalles [240-350] kg et [72-96] mois. Le poids des fœetus a été compris entre 47 et $5600 \mathrm{~g}$. Toutes les gestations utilisées dans la présente expérience ont été simples et complètes.

\section{Biométrie}

Les examens ont été réalisés par un opérateur expérimenté en bain d'eau au moyen d'un échographe (Chison Medical Imaging Co., Ltd., 8300) équipé d'une sonde convexe de 3,5 MHz. Chaque appareil gravide a été plongé dans une bassine d'eau selon la méthode décrite par Zongo et al. (2014) avec de légères modifications. La sonde, tenue à la main du technicien, a été ensuite immergée dans l'eau et orientée en direction de l'utérus gravide. L'opérateur effectue des mouvements de rotation autour de la corne gravide afin de visualiser les différents organes du foetus. Le pouvoir de résolution de la sonde est de $2 \mathrm{~mm}$. Les images vidéo des structures fotales ont été projetées sur l'écran puis gelées afin de prendre les dimensions à l'aide d'un instrument métrique intégré à l'appareil. L'utérus a ensuite été disséqué, le fotus recueilli et pesé avec une balance Kem 440-53N de précision de $1 \mathrm{~g}$. Les paramètres fœtaux examinés ont été les DA, DBP, DCO, la DCU, et les LD, LF, LT, et le poids. Les dimensions biométriques relevées ont été corrélées avec les poids des foetus mesurés à la balance.

Le DA à l'échographie a été défini comme la distance comprise entre les deux extrémités de l'image de la coupe transversale de l'abdomen (fig. 1). Le DBP a été le diamètre de l'image de la coupe transversale des deux pariétaux projetés sur l'écran (fig. 1). Cette distance a été mesurée à partir de la marge externe d'un os pariétal à l'autre comme définie par Hildebrandt et al. (2007).

Le DCO a été défini comme le diamètre de la coupe transversale du cordon ombilical projeté sur l'écran (fig. 1) et le DCU comme la distance comprise entre les deux extrémités de l'image de la corne utérine gravide (fig. 1) comme décrit par Zongo et al. (2014).

Les LF et LT ont été mesurées en fonction des zones de calcifications intensives qui semblaient riches en échos (Abdelghafar et al. 2012). Les LF et LT ont été respectivement définies comme la distance entre les protubérances des deux extrémités du fémur et du tibia (fig. 1). La LD a été définie comme la distance entre l'éminence de la nuque et l'articulation du sacrum-coccygène (Nwaogu et al. 2010; Zongo et al. 2014) (fig. 1).

\section{Analyses statistiques des données}

Les données collectées ont été exprimées en moyennes \pm écarts-types et analyser au moyen du logiciel $\mathrm{R}$ (Windows, version 3.4.2). La normalité de la distribution des données et des résidus a été établie avec le test de Shapiro (Shapiro-Wilk test). Un nuage de points a été généré et ajusté avec une courbe de tendance qui rapporte au mieux le modèle de régression du paramètre mesuré avec le poids. Les équations de ces courbes qui constituent les formules d'estimation du poids du fotus ont été générées à partir de la fonction $\operatorname{lm}(\log (y \sim x))$. Les coefficients de détermination $\left(R^{2}\right)$ et de corrélation $(R)$ ont été également déterminés par cette fonction. Les niveaux de prédiction du poids du fotus à partir des mesures des paramètres 
Fig. 1. Images échographiques des mensurations réalisées en bain d'eau chez le fotus zébu peulh. [Couleur en ligne.]
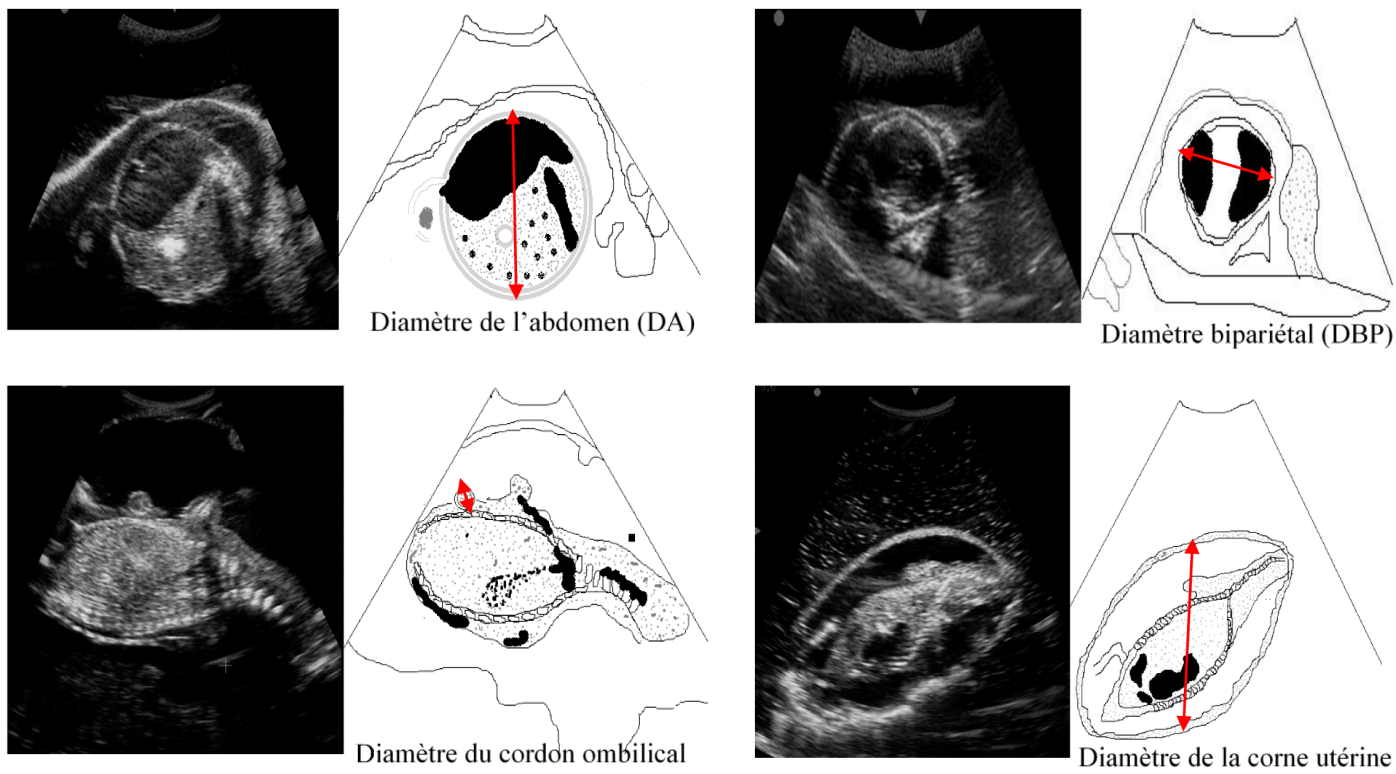

Diamètre du cordon ombilical
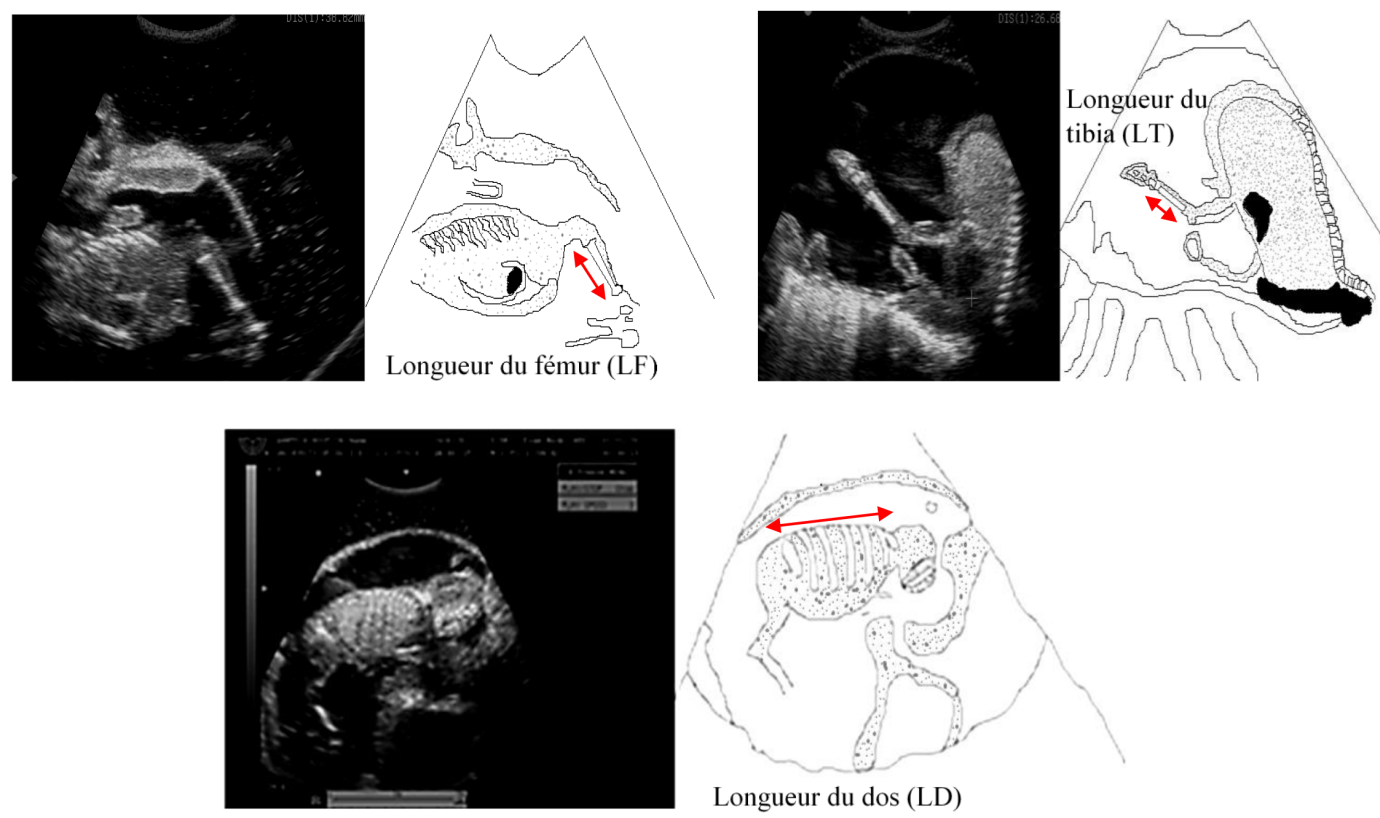

biométriques ont été évalués par le pourcentage des coefficients de détermination. Les résultats ont été considérés significatifs au seuil de probabilité de $5 \%$.

\section{Résultats}

Estimation du poids du fotus zébu à partir des structures fotales

Les courbes de tendance optimales qui rendent compte au mieux la relation entre les paramètres considérés et le poids du fœtus ont été celles du modèle de régression curviligne (fig. 2). La dispersion des nuages de points autour des courbes de régression semble varier en fonction de la dimension et du paramètre mesuré (fig. 2). Les équations qui décrivent au mieux cette relation ont été de type $y=a x^{b}$, où " $y$ » représente le poids du fotus

estimé en gramme ; « $x$ » représente le paramètre fotal mesuré en millimètre ; $a$ et $b$ sont des constantes (fig. 2).

Toutes les structures biométriques (DA, DBP, DCO, DCU, LDO, LF, et LT) considérées dans la présente étude ont présenté des corrélations positives et hautement significatives avec le poids du fœtus $(p=0,0001)$ (tableau 1). Les paramètres biométriques qui présentent la plus forte corrélation $(R \geq 0,90)$ avec le poids ont été le DA $(R=0,97)$ et la $L F(R=0,90)$ (tableau 1). Le DCO et le DCU ont présenté des corrélations similaires avec le poids du fotus $(R=0,82)$. Le degré de corrélation entre le poids, la LT, le DBP et la LD a été rapporté dans le tableau 1 . Le DA, le DBP, et la LF semblent être les meilleurs estimateurs du poids du fotus (fig. 2). Les niveaux de prédiction du poids fœtal à 
Fig. 2. Courbes d'évolution du poids du fœtus à partir des mesures échographiques des paramètres morphologiques fœetaux. [Couleur en ligne.]
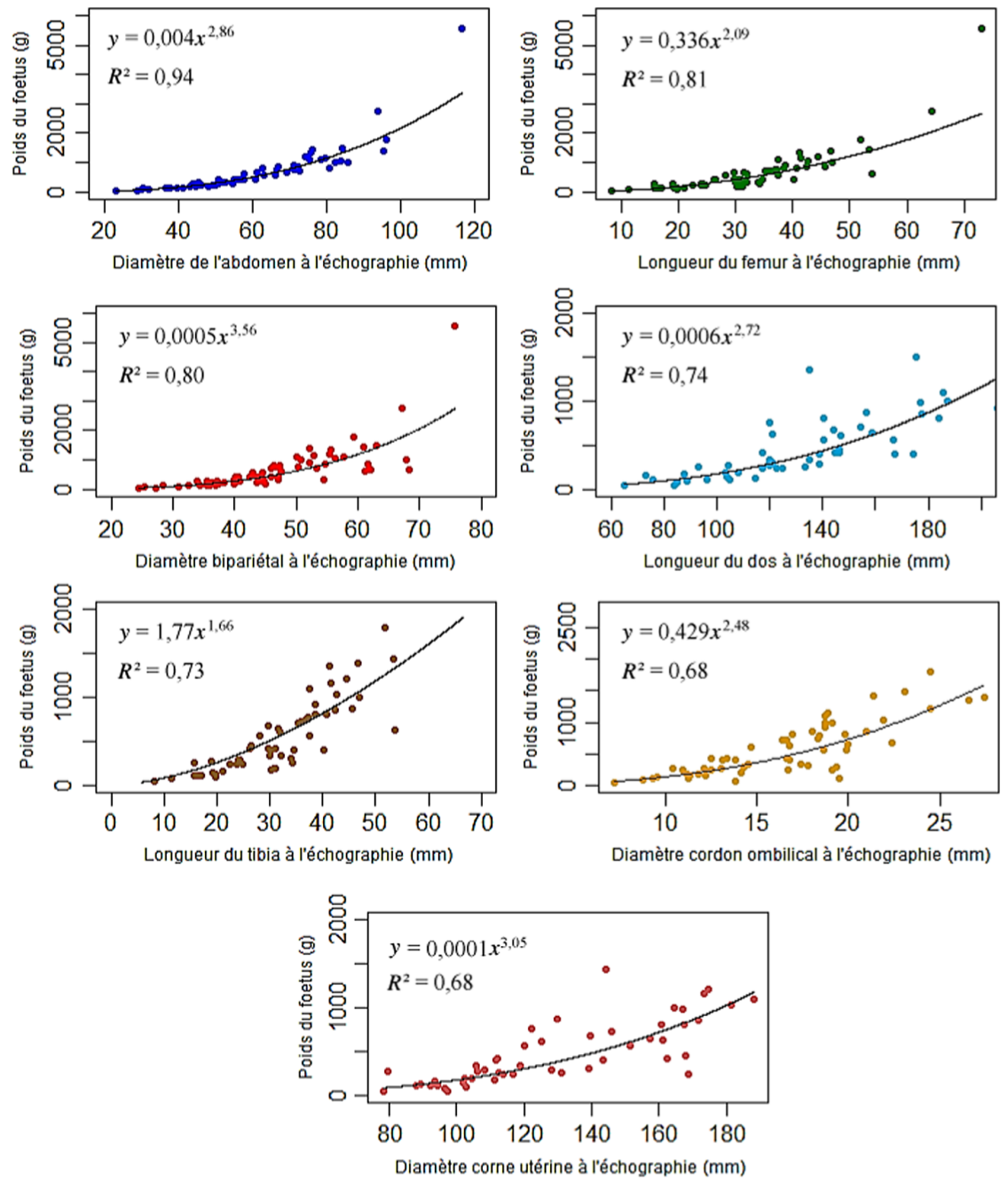

Tableau 1. Relations entre les paramètres biométriques étudiées et le poids du fœetus zebus.

\begin{tabular}{llll}
\hline & $\begin{array}{l}\text { Nombre de } \\
\text { foetus }(N)\end{array}$ & $\begin{array}{l}\text { Corrélation }(R) \text { avec le } \\
\text { poids du fotus }\end{array}$ & $P$ \\
\hline Diamètre de l'abdomen & 61 & 0,97 & $* * *$ \\
Longueur du femur & 61 & 0,90 & $* * *$ \\
Diamètre bipariétal & 61 & 0,89 & $* * *$ \\
Longueur du dos & 61 & 0,86 & $* * *$ \\
Longueur du tibia & 61 & 0,85 & $* * * *$ \\
Diamètre du cordon ombilical & 61 & 0,82 & $* * *$ \\
Diamètre de la corne utérine & 61 & 0,82 &
\end{tabular}

Note: ${ }^{*}, p<0,05 ;{ }^{* *}, p<0,01 ; * * *, p<0,001$.

partir des LD, du LT, des DCO, et de la DCU ont été de l'ordre de 74,73 , et $68 \%$ respectivement (fig. 2 ).

\section{Discussion}

La plupart des données disponibles sur la physiologie du développement fœtal des bovins se rapportent à l'endocrinologie et à l'organogénèse (Szenci et al.
1998; Tainturier et al. 2000). Ces données portant sur l'organogénèse ont permis d'établir des formules d'estimation de l'âge gestationnel en fonction du paramètre biométrique mesuré (Lamb et Fricke 2004). Le suivi de la croissance fotale à travers les performances pondérales est d'application courante chez les petits ruminants et des rongeurs (Carr et al. 2011; Nguyen et al. 2012; 
Zongo et al. 2014). Chez les bovins, cet aspect du développement fotal est jusqu’à nos jours peu envisagé. Le suivi de la croissance fotale permet, chez les humains, d'anticiper la voie d'accouchement et de réduire le taux de mortalité embryonnaire et néo-natale (McCallum et Brinkley 1979; Jordaan 1983; Hadlock et al. 1985).

Dans la présente étude, l'estimation du poids du fotus zébu en fonction des mesures échographiques des paramètres fotaux, a consisté à apprécier la corrélation existante entre les DA, DBP, DCO, DCU, les LF, LT, LD et le poids. Ces paramètres ont tous présenté des corrélations positives et hautement significatives avec le poids du fotus chez les caprins, ovins et des rongeurs (Carr et al. 2011; Nguyen et al. 2012; Zongo et al. 2014). En médicine humaine, ils sont utilisés dans les formules d'estimation du poids fœtal (Hadlock et al. 1984; Kumara et Perera 2009; Perdriolle-Galet et al. 2014). Ces différents paramètres, outre la forte corrélation qui les lient au poids du fotus zébu, décrivent avec ce dernier, un modèle de croissance de type curvilinéaire. Ce modèle de croissance a été par ailleurs rapporté dans la description de la corrélation existante entre le poids du fotus caprin et ces paramètres ci-dessus cités. Chez les ovins, le poids du fœtus décrit le même modèle de croissance avec la LD et la circonférence du cœur entre le 50 et 134 jour de la gestation (Neville et al. 2008). Cependant, ce modèle contraste avec le modèle linéaire rapporté dans les formules d'estimation du poids fotal humain (Hadlock et al. 1984).

Le DA, la LF, et le DBP ont été les plus appropriés pour l'estimation du poids du fotus zébu. D’après Carr et al. (2011), la partie abdominale est le lieu de stockage de matières grasses sous-cutanées. Chez l'espèce humaine, c'est le meilleur marqueur de la croissance fotale (Degani 2001). L'abdomen du fotus a été accessible et facile à mesurer au cours des examens échographiques. Ce qui pourrait expliquer la forte corrélation du DA avec le poids du fœetus zébu. La LF explique respectivement 63 et $66 \%$ la variation du poids du fotus ovin et caprin (Carr et al. 2011; Zongo et al. 2014). Chez le fotus humain, il ressort l'existence d'une forte corrélation entre la LF et la longueur couronne-talon. La longueur couronnetalon, serait fortement corrélée avec le poids du fœtus humain (McCallum et Brinkley 1979; Jordaan 1983). Cette relation entre la LF et la couronne-talon serait probablement variable en fonction de l'espèce étudiée. Cette tendance pourrait probablement être la cause de cette différence observée entre les données de la présente étude et des résultats rapportés chez les caprins, ovins et les rongeurs. Chez les ovins, la dynamique de la croissance fœtale s'explique à hauteur de $99 \%$ avec le DBP (Vural et al. 2008). Chez le fotus caprin et ovin, le poids se prédit respectivement à 87 et $45 \%$ avec ce paramètre (Zongo et al. 2014; Carr et al. 2011).

Le niveau d'estimation du poids du fœtus zébu à partir de la LD, la LT, le DCO et le DCU est légèrement moins élevé $\left(R^{2}<0,75\right)$ comparativement à celui rapporté avec les DA, DBP et la LF $\left(R^{2} \geq 0,89\right)$. Le regroupement des nuages de points autour des courbes de tendances optimales décrivant la relation entre ces paramètres et le poids fotal dénote une forte sensibilité de ces structures morphologiques à prédire le poids du fœtus zébu aux premiers stades de la gestation. Des résultats similaires ont été également rapportés chez les caprins (Zongo et al. 2014). Ces paramètres, excepté la LT, ne sont pas d'applications courantes dans l'estimation du poids du fotus humain (Hadlock et al. 1984). Chez les bovins, compte tenu de la profondeur des organes génitaux et de la position du fotus aux stades tardifs de la gestation, ces paramètres pourront servir d'alternative aux DA, LF et DBP à l'estimation du poids.

\section{Conclusion}

Ces données sont les premiers résultats à rapporter in vitro, l'estimation du poids du fœtus zébu en fonction de la biométrie des organes. Cette approche échographique est une méthode importante au service des techniciens et des vétérinaires dans le contrôle de la reproduction du zébu. Les paramètres les plus appropriés pour l'estimation du poids ont été les DA $\left(y=0,004 x^{2,86}\right)$, DBP, et la LF. Ces résultats sont de précieux repère qui permettent de prendre des décisions qui contribuent à résoudre les complications liées à la mise bas chez les femelles zébu locales. Ils constituent en outre, des données manquantes dans la littérature scientifique sur la reproduction du zébu. Toutefois, pour une exploitation optimale de cette étude en milieu réel, il est important de s'assurer d'une contention adéquate des animaux afin de garantir une manipulation aisée de l'opérateur.

\section{Remerciements}

Cette étude est un travail introductif à l'application de l'échographie sur la reproduction du zébu peulh en zone soudano-sahélien. Ce travail a été financé par la Fondation internationale pour la science à travers le Boursier B/3281-2. L'Agence internationale de l'énergie atomique (BKF2012004) a fourni des équipements pour le laboratoire. Les auteurs adressent à ces deux institutions leurs sincères remerciements pour leur appui technique et financier dans la réalisation de cette étude. Que ces institutions trouvent ici, la marque de notre profonde gratitude.

\section{References}

Abdelghafar, R.M., Ahmed, B.H., Abdelrahim, S.M., and Ibrahim, M.T. 2012. The accuracy of gestational age predicted from femur and humerus length in Saanen goats using ultrasonography. Acta Vet. Brno. 81: 295-299. doi:10.2754/ avb201281030295.

Azevedo, E.M.P., Aguiar, C.R.F., Freitas, L.M.N, Rabelo, M.C., Santos, M.H.B., Lima, P.F., et al. 2007. Ultrasound fetal measurement parameters for early estimate of gestational age and birth weight in ewe. Med. Vet. Rec. 2: 56-61.

Branum, A.M., and Schoendorf, K.C. 2003. The effect of birth weight discordance on twin neonatal mortality. Obstet. 
Gynecol. 101(3): 570-574. doi:10.1016/s0029-7844(02)03119-8. PMID:12636964.

Carr, D.J., Aitken, R.P., Milne, J.S., David, A.L., and Wallace, J.M. 2011. Ultrasonographic assessment of growth and estimation of birth weight in late gestation fetal sheep. Ultrasound Med. Biol. 10: 1588-1595. doi:10.1016/j.ultrasmedbio.2011.06.016.

Craig, M., Zelig, M.D., Shad, H., Deering, M.D., Peter, G., and Napomitano, M.D. 2009. Improved ultrasonographic estimation of birth weight in macrosomic fetuses by application of a correction factor to the gestation-adjusted projection method. J. Ultrasound Med.. 28: 1357-1364.

Degani, S. 2001. Fetal biometry: clinical pathological and technical considerations. Obstet. Gynecol. 56: 159-167. doi:10.1097| 00006254-200103000-00023

Elliott, C., Morton, J., and Chopin, J. 2009. Factors affecting foal birth weight in Thorough bred horses. Theriogenology, 71: 683-689. doi:10.1016/j.theriogenology.2008.09.041. PMID:18980778.

Hadlock, F.P., Harrist, R.B., Carpenter, R.J., Deter, R.L., and Park, S.K. 1984. Sonographic estimation of fetal weight. The value of femur length in addition to head and abdominal measurements. Radiology, 150: 535-540. doi:10.1148/radiology.150. 2.6691115. PMID:6691115.

Hadlock, F.P., Harrist, R.B., Sharman, R.S., Deter, R.L., and Park, S.K. 1985. Estimation of fetal weight with the use of head, body, and femur measurements-a prospective study. Am. J. Obstet. Gynecol. 151: 333-337. doi:10.1016/0002-9378(85) 90298-4. PMID:3881966.

Hildebrandt, T., Drews, B., Gaeth, A.P., Goeritz, F., Hermes, R., Schmitt, D., and Gray, C. 2007. Fetal age determination and development in elephants. Proc. R. Soc. B, 274: 323-331. doi:10.1098/rspb.2006.3738. PMID:17164195.

Ivars, J., Houfflin-Debarge, V., Vaast, P., and Deruelle, P. 2010. Précision de l'estimation du poids fotal par l'échographie dans les grossesses gémellaires. Gynecol. Obstet. Fertil. 38: 740-746. doi:10.1016/j.gyobfe.2010.10.001.

Jordaan, H.V.F. 1983. Estimation of fetal weight by ultrasound. J. Clin. Ultrasound, 11: 59-66. doi:10.1002/jcu.1870110202. PMID:6404938.

Kumara, D.M.A., and Perera, H. 2009. Evaluation of six commonly used formulae for sonographic estimation of fetal weight in a Sri Lankan population. Sri Lanka J. Obstet. Gynaecol. 31: 20-33. doi:10.4038/sljog.v31i1.1735.

Lackman, F., Capewell, V., Richardson, B., da Silva, O., and Gagnon, R. 2001. The risks of spontaneous preterm delivery and perinatal mortality in relation to size at birth according to fetal versus neonatal growth standards. Am. J. Obstet. Gynecol. 184: 946-953. doi:10.1067/mob.2001.111719. PMID:11303203.

Lafont, M., Dellinger, P., Mutumba, W., Bernard, C., and Hoyek, T. 2016. Accuracy of ultrasound estimated fetal weight at term. Gynecol. Obstet. Fertil. 44: 391-395. doi:10.1016/ j.gyobfe.2016.05.005. PMID:27426690.

Lamb, G.C., and Fricke, P.M. 2004. Ultrasound - early pregnancy diagnosis and fetal sexing. Appl. Reprod. Strateg. Beef Cattle, 219-229. [Online]. Available from https://www.researchgate.net/ publication/267236571_ULTRASOUND_-_EARLY_PREGNANCY_ DIAGNOSIS_AND_FETAL_SEXING.
McCallum, W.D., and Brinkley, J.F. 1979. Estimation of fetal weight from ultrasonic measurements. Am. J. Obstet. Gynecol. 133: 195-200. doi:10.1016/0002-9378(79)90475-7. PMID:420274.

Neville, T.L., Ward, M.A., Reed, J.J., Soto-Navarro, S.A., Julius, S.L., and Borowicz, P.P., 2008. Effects of level and source of dietary selenium on maternal and fetal body weight, visceral organ mass, cellularity estimates, and jejunal vascularity in pregnant ewe lambs. J. Anim. Sci. 86: 890-901. doi:10.2527| jas.2006-839. PMID:18192546.

Nguyen, T.M., Nakamura, H., Wakabayashi, A., Kanagawa, T., Koyama, S., Tsutsui, T., et al. 2012. Estimation of mouse fetal weight by ultrasonography: application from clinic to laboratory. Lab. Anim. 46: 225-230. doi:10.1258/la.2012.011117. PMID:22723651.

Nwaogu, I.C., Anya, K.O., and Agada, P.C. 2010. Estimation of fetal age using ultrasonic measurements of different fetal parameters in red Sokoto goats (Capra hircus). Vet. Arch. 2: 225-233. [Online]. Available from https://hrcak.srce.hr/file/85781.

Perdriolle-Galet, E., Thiebaugeorges, O., Lamy, C., Makke, L., Barbier, A., Monceau, E., and Morel, O. 2014. Estimation du poids fotal en salle de naissance : performances respectives de la clinique et de l'échographie. J. Gynecol. Obstet. Biol. Reprod. 43: 593-599. doi:10.1016/j.jgyn.2013.08.016.

Pitala, W., Zongo, M., Boly, H., Sousa, N.M., Sawadogo, L., Leroy, P., et al. 2012. Application de l'échographie en reproduction chez le zébu Goudali. Int. J. Biol. Chem. Sci. 5: 2055-2065. doi:10.4314/ijbcs.v6i5.15.

Robinson, J.J. 1977. Studies on reproduction in prolific ewes. I. Growth of the products of conception. J. Agric. Sci. 88: 539-552. doi:10.1017/S0021859600037229.

Szenci, O., Taverne, M.A.M., Beckers, J.F., Sulon, J., Varga, J., Borzsonyi, L., et al. 1998. Evaluation of false ultrasonographic diagnoses in cows by measuring plasma levels of bovine pregnancy-associated glycoprotein 1 . Vet. Rec. 142: 304-306. doi:10.1136/vr.142.12.304. PMID:9569497.

Tainturier, B., Tainturier, D., and Bencharif, D. 2004. Fetal sex determination in cattle by ultrasonography. J. Anim. Vet. Adv. 3: 136-141.

Tainturier, D., Fieni, F., Bruyas, J.F., Battut, I., and Bencharif, D. 2000. Diagnostic de gestation chez la vache par échotomographie : application au diagnostic du sexe. Giornate buiatriche, Asiago, Italy, 5, 6 et 7 mai 2000. Vol. 32, pp. 63-68.

Vieira, P.S., Nogueira, C.E.W., Santos, A.C., Borba, L.A., Scalco, R., Brasil, C.L., et al. 2018. Development of a weight-estimation model to use in pregnant criollo-type mares. Cienc. Rural. 48: 1. [Online]. Available from http://www.scielo.br/scielo. php?script=sci_arttext\&pid=S0103-84782018000100701.

Vural, M.R., Sel, T., Karagul, H., Ozenc, E., Orman, M., Izgur, H., and Kuplulu, S. 2008.Ultrasonographic examinations of embryonic-fetal growth in pregnant Akkaraman ewes fed selenium supply and dietary selenium restriction. Rev. Med. Vet. 159: 628-633. [Online]. Available from https:// www.revmedvet.com/2008/RMV159_628_633.pdf.

Zongo, M., Traoré, I., Pitala, W., Boly, H., Sanou, D., Belemtougri, R., and Sawadogo, L. 2014. Estimation du poids du fotus de la chèvre sahélienne à partir des mesures échographiques des paramètres fotaux. Can. J. Anim. Sci. 94: 437-443. doi:10.4141/cjas-2014-050. 